\title{
Bosentan Inducing Autoimmune Hepatitis in a Patient with Idiopathic Pulmonary Arterial Hypertension
}

\author{
Alexandre de Araujo ${ }^{1}$, Augusto Mantovani ${ }^{1}$, Carlos Thadeu Schmidt Cerski ${ }^{2}$, Antonio Barros Lopes ${ }^{1}$, Luiza Cristina \\ Bortoncello $^{1}$, Marcelo Basso Gazzana ${ }^{3}$, Hugo Cheinquer ${ }^{1}$
}

1) Gastroenterology and

Hepatology Division,

2) Pathology Division,

3) Pneumology Division,

Hospital de Clínicas de Porto

Alegre,

Porto Alegre, Brazil

Address for correspondence: Augusto Mantovani, MD

Hospital de Clínicas de Porto Alegre,

2350 Ramiro Barcelos Street; Porto Alegre - RS, 90035-007, Brazil augustomanto@gmail.com

Received: 25.08.2017

Accepted: 30.11.2017

\section{ABSTRACT}

A 41-year-old woman diagnosed with idiopathic pulmonary hypertension presented symptoms despite the use of vasodilators, requiring treatment with bosentan. Previously, the patient had no signs of autoimmunity and had normal liver function. After three years of bosentan use, aminotransferase levels increased, without improvement after bosentan suspension, leading to complementary investigation. The diagnosis of autoimmune hepatitis was confirmed by biopsy, already in the stage of cirrhosis. In conclusion, in case of aminotransferase levels that remain persistently elevated, despite the reduction in doses and/or suspension of bosentan, autoimmune hepatitis must be investigated and treated urgently due to possibly rapid progression to cirrhosis..

Key words: Endothelin receptor antagonists - autoimmunity - hepatotoxicity - cirrhosis - liver biopsy.

Abbreviations: AIH: Autoimmune hepatitis; ALP: Alkaline phosphatase; ALT: Alanine aminotransferase; ANA: Antinuclear antibodies; ASMA: Anti-smooth muscle antibody; AST: Aspartate aminotransferase; ERA: Endothelin receptor antagonist; GGT: Gamma-glutamyltransferase; HAV: Hepatitis A virus; HBV: Hepatitis B virus; HCV: Hepatitis C virus; HIV: Human immunodeficiency virus; MRI: Magnetic resonance imaging; PAH: Pulmonary arterial hypertension; RV: Right ventricle; RVEF: Right ventricle ejection fraction.

\section{INTRODUCTION}

Hepatotoxicity is a wellknown adverse event in patients treated with endothelin receptor antagonists (ERAs), such as bosentan. These drugs are reported to inhibit bile salt export pumps, which causes intracellular bile salt accumulation in hepatocytes [1]. When hepatic damage occurs, the recommendation is to decrease the dose of bosentan and to observe the evolution of the aminotransferases. However, when there is no improvement in liver function despite the discontinuation of the drug in question, an underlying liver disease may be present [2].

Herein, we present the case of a female patient with a long history of idiopathic pulmonary arterial hypertension (PAH), with no evidence of liver disease or associated autoimmune condition, who developed autoimmune hepatitis (AIH) after three years of treatment with bosentan, already presenting with cirrhosis at the biopsy to confirm the etiology of her condition.

\section{CASE REPORT}

A 41-year-old woman with a ten-year history of progressive exertional dyspnea, poorly controlled with initial measures, was referred to the Pulmonary Hypertension outpatient clinic at Hospital de Clínicas de Porto Alegre in October 2009. The hypothesis of idiopathic $\mathrm{PAH}$ was confirmed by a right heart catheterization demonstrating a mean pulmonary arterial pressure of $61 \mathrm{mmHg}$, pulmonary arterial occlusion pressure of $6 \mathrm{mmHg}$, cardiac output of $4.4 \mathrm{~L} / \mathrm{min} / \mathrm{m}^{2}$ and pulmonary vascular resistance of $12.5 \mathrm{WU}$. The lung scan showed no perfusion defects, thoracic computed angiotomography did not show signs of interstitial disease, the antinuclear antibodies (ANA), lupus anticoagulant and rheumatoid factor were negative, and collagen vascular diseases were excluded. There were no other clinical findings suggestive of secondary pulmonary hypertension. Human immunodeficiency virus (HIV), hepatitis B and C serology, anti-smooth muscle antibody (ASMA) were all negative. At the initial evaluation, aspartate (AST) and alanine aminotransferases (ALT), bilirubin, alkaline 
phosphatase (ALP) and gamma-glutamyltransferase (GGT) were all within the normal range.

Initially the patient was treated with sildenafil and warfarin, with a partial improvement in exertional dyspnea, at this time with class II modified New York Heart Association (NYHA). In March 2013, bosentan (62.5mg BID for 4 weeks and then $125 \mathrm{mg}$ BID as maintenance) was associated in the therapeutic regimen due to a worsening dyspnea (class III modified NYHA), with a partial clinical response and maintenance of the normal level of aminotransferases.

The patient was referred to the pulmonary transplantation outpatient clinic; but in November 2014, she opted not to continue the evaluation, despite having been advised about the severity of her disease, risk of progression and symptomatic worsening and death. In January 2015, due to chronic desaturation and persistence of symptoms, home oxygen therapy was indicated.

The patient had underwent cardiac magnetic resonance imaging (MRI) in April 2014, demonstrating a normal left ventricular systolic function; the right ventricle (RV) was dilated and with moderate systolic dysfunction (right ventricle ejection fraction, RVEF, of $30 \%$ ), with marked dilatation of the pulmonary artery trunk. After prolonged use of bosentan, she repeated the cardiac MRI in August 2015 - demonstrating a RVEF 54\% with normalization of RV systolic function, and then in November 2016, with a new improvement in RV systolic function and reduction of systolic bulging of the interventricular septum to the left, indicating a reduction of the RV overload.

From the initiation of the treatment with bosentan until July 2016, the levels of aminotransferases, bilirubin, ALP and GGT remained within the normal range. In August 2016, the laboratory tests began to alter: AST $223 \mathrm{IU} / \mathrm{L}$, ALT $250 \mathrm{IU} / \mathrm{L}$, total bilirubin $1.3 \mathrm{mg} / \mathrm{dL}$, conjugated bilirubin $0.8 \mathrm{mg} / \mathrm{dL}$, ALP $161 \mathrm{IU} / \mathrm{L}$ and GGT $411 \mathrm{IU} / \mathrm{L}$.

The patient returned on December 13th 2016, claiming nausea and postprandial fullness, presenting jaundice, with a bilirubin of $3.1 \mathrm{mg} / \mathrm{dL}$, conjugated bilirubin of $2.6 \mathrm{mg} / \mathrm{dL}$, AST 258 IU/L, ALT 188 IU/L, ALP 118 IU/L, GGT 157 IU/L; serology for HIV, HCV, HAV and HBV remained all negative. At this consultation, the bosentan dose was halved. The patient returned two weeks later, still presenting jaundice and altered laboratory tests. At this point, bosentan was discontinued and the patient was admitted to our hospital for additional investigations.

The inpatient laboratory showed a positive ANA result (1/640 - dense fine speckled nuclear pattern and speckled cytoplasmic pattern), IgG of $3204 \mathrm{mg} / \mathrm{dL}$, anti-smooth muscle antibody positive (1/320) and an abdominal Doppler ultrasonography with signs of chronic liver disease, without portal or hepatic vein thrombosis.

A transcutaneous hepatic biopsy was performed showing chronic hepatitis with intense activity, cirrhosis, lymphoplasmocytic infiltrate, hepatocellular rosette formation, an Ishak activity score of $3+2+0+3(8 / 18)$ and a METAVIR score of A3F4 (Figs. 1-4). The diagnosis of AIH leading to cirrhosis was confirmed, probably induced by bosentan, and the patient was started on azathioprine and prednisone. Two months later, a complete laboratory response was observed (Fig. 5).

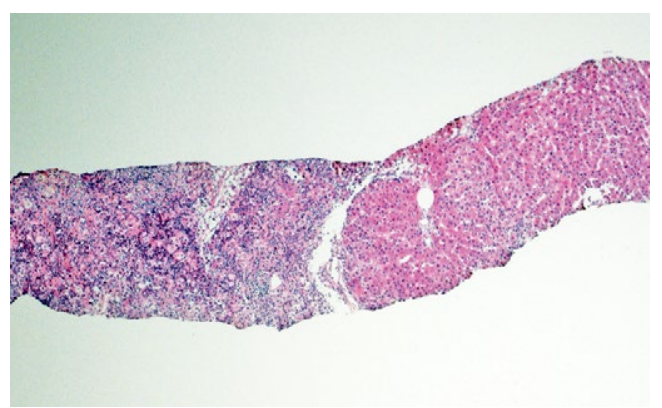

Fig. 1. Liver biopsy showing parenchyma distortion consistent with cirrhosis (H\&E, x4).

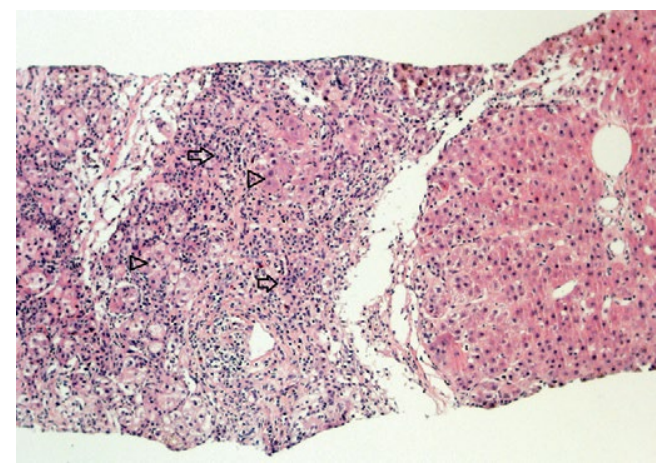

Fig. 2. Liver biopsy with typical autoimmune hepatitis findings: lymphoplasmocytic infiltrate (arrows) and hepatic rosette formation (arrow heads) (H\&E, x10).

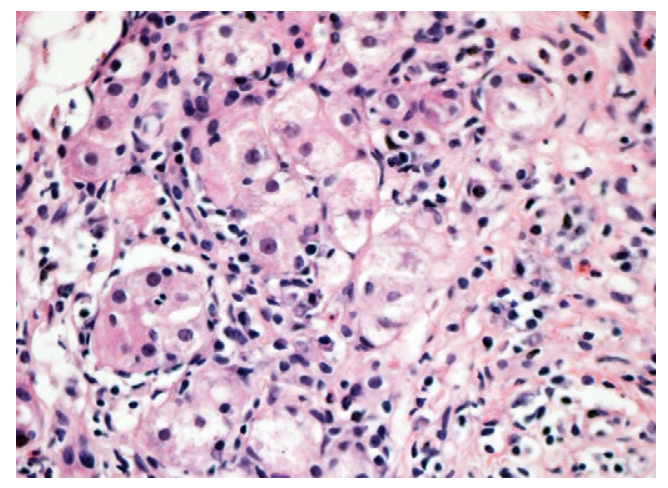

Fig. 3. Hepatic rosette formation seen in liver biopsy specimen (H\&E, $x 400)$.

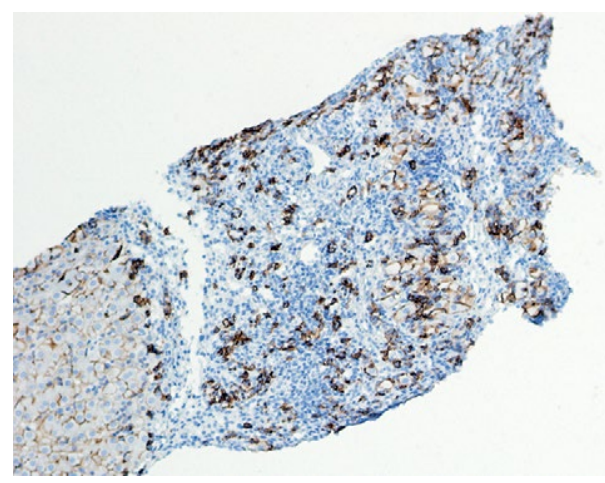

Fig. 4. Immunohistochemical stain showing lymphocytic infiltrate (CD 138 stain, x10). 


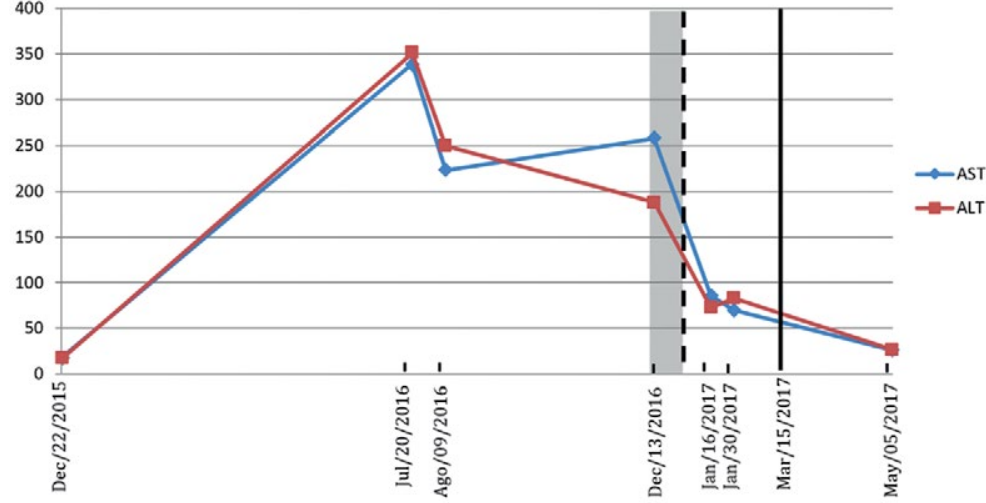

Fig. 5. Aspartate aminotransferase (AST) and alanine aminotransferase (ALT) results from December/2015 until May/2017. The grey zone (starting in December 14th, 2016) is the period when the patient received the half dose of bosentan. The dashed vertical line shows when bosentan was suspended (December 27th, 2016). Azathioprine $50 \mathrm{mg}$ and prednisone $30 \mathrm{mg}$ were introduced in March/2017 (solid vertical line).

\section{DISCUSSION}

Autoimmune hepatitis is a chronic self-perpetuating inflammatory disease that may start with an episode of acute hepatitis and may lead to liver cirrhosis, liver cancer, liver transplantation or death. Its diagnosis is often challenging and based on the clinical aspect, elevation of ALT, AST and gammaglobulins - in particular serum IgG levels, autoantibodies and histology [3].

The patient of this report had all the hallmarks of AIH and was diagnosed based on The Revised International Autoimmune Hepatitis Group Modified Scoring System with a pre-treatment score of 16 (classified as definite AIH) [4].

Due to the elevation of aminotransferase levels during therapy with bosentan, initially we suspected drug-induced hepatotoxicity. However, because of the slow descent pattern of the aminotransferases, even after we discontinued bosentan, and as we proceeded with the investigation, we had reason to believe that bosentan had acted as an autoimmunity trigger.

The liver biopsy was performed to clarify the etiology between drug-induced liver injury and AIH. Liver biopsy revealed disarrayed hepatocytes and a lymphoplasmocytic infiltrate, characteristic of an interface hepatitis, with a hepatic rosette pattern of regeneration. With this finding, the diagnosis of AIH was sealed and the patient started immunosuppressive treatment.

The hepatotoxicity of bosentan is well described in the literature, including in the manufacturer's labeling. But only two cases of bosentan acting as an inducer of AIH have been described. One was in a pediatric study of bosentan for treatment of $\mathrm{PAH}$, in which 33 patients were enrolled and one case of AIH was reported [5]. A previously bosentan-naïve patient, who was receiving treatment for 9 months, showed an increase in liver enzymes which remained elevated following a subsequent dose reduction of bosentan, presented F-actin antibody and ASMA positive. The drug was interrupted and the patient showed improvement in follow-up testing, so bosentan was resumed. However, a few days later the liver enzymes increased again, which led to the permanent discontinuation of bosentan.

In the adult population, a case was described by Naito et al. [6], in which a 48-year-old woman was receiving bosentan for a period of four years when she presented drug-related hepatotoxicity. Bosentan was replaced by ambrisentan, another ERA but, after a year and a half, a second episode of hepatic dysfunction occurred, with no improvement after discontinuation of medication, leading the team to further investigation with a subsequent diagnosis of AIH. Sitaxentan, another representative of the ERA class, was associated with exacerbation of a previously diagnosed AIH in a patient with $\mathrm{PAH}$ secondary to systemic sclerosis in Germany [7]. This patient had been using sitaxentan for 14 months and presented an important increase of the aminotransferases; ambrisentan was added to the therapeutic regimen but was not clinically tolerated, and also increased the aminotransferase levels. It was then attempted to return sitaxentan to the prescription, but after 10 days of treatment, aminotransferases increased once again in association with an increased IgG level. In this regard, sitaxentan was withdrawn from the worldwide market following information on two cases of fatal liver injury [8].

The most well-known explanation for bosentan hepatotoxicity is the inhibition of the hepatocanalicular bile salt export pump, leading to cholestatic damage by intrahepatic bile salts accumulation. However, in a study using a sandwichcultured human hepatocytes (representing liver, blood and bile), a new metabolite of bosentan was identified during CYP2C9 metabolism, with decreased hepatocyte viability due to the role on the t-butyl group hydroxylation of bosentan [9].

In contrast to the increasingly elucidated bosentan hepatotoxicity, its use was shown to be beneficial in hepatic microcirculation in cirrhotic rats after intraperitoneal injection of Escherichia coli endotoxin. This study demonstrated the inhibitory action of bosentan in the up-regulation of endothelin, inducible nitric oxide synthase and cyclooxygenase-2 mRNA expressions, preventing hepatocellular damage during endotoxemia in cirrhotic rats [10].

Our case is interesting because the patient had normal liver function, no laboratory exams suggesting an autoimmune pattern and did not use another ERA before or during bosentan treatment. In the Japanese case, the patient already had ANA positive (1/1280) and anti-centromere antibodies, suggesting some autoimmune activity, before the treatment with any medication for $\mathrm{PAH}$, and in the German case the patient already had AIH previously diagnosed and the ERA acted as an exaggerator. Another notable aspect is the advanced degree of liver damage, presenting with cirrhosis, with abdominal imaging exams a few years before showing absence of any liver disease.

Although the patient's underlying disease may progress to right heart failure - leading to sinusoidal dilation, hepatic congestion, and finally fibrosis - the biopsy performed in 
our patient demonstrated several characteristics compatible with AIH, with no evidence of sinusoidal dilatation, chronic congestion and no specific alterations around the central lobular hepatic vein (zone 3 ).

\section{CONCLUSION}

It is not possible to state that the patient in question developed AIH due to the use of bosentan. However, as there are reports of exacerbation of previously diagnosed $\mathrm{AIH}$ and a case report of its development after the onset of ERA use, it is possible to infer that there might be an association between the development of AIH and the use of bosentan.

We recommend that in patients with persistently elevated aminotransferase levels, despite the reduction and/ or suspension of bosentan, autoimmune hepatitis must be considered and treated urgently due to possible rapid progression to cirrhosis.

Conflicts of interest: The authors declare that there is no potential conflict of interest relevant to this article. Grants and financial support: none.

Authors' contribution: A.A., A.M., A.B., H.C., M.B.G. and L.C.B. managed the patient and contributed to the diagnosis, follow-up and the writing of the manuscript. C.T.S.C. performed the histological analysis and work-up and the photo documentation. All authors contributed to the writing of the manuscript.

\section{REFERENCES}

1. Fattinger K, Funk C, Pantze M, et al. The endothelin antagonist bosentan inhibits the canalicular bile salt export pump: a potential mechanism for hepatic adverse reactions. Clin Pharmacol Ther 2001;69:223-231. doi:10.1067/mcp.2001.114667
2. Galiè N, Corris PA, Frost A, et al. Updated treatment algorithm of pulmonary arterial hypertension. J Am Coll Cardiol 2013;62:D60-D72. doi:10.1016/j.jacc.2013.10.031

3. Manns MP, Lohse AW, Vergani D. Autoimmune hepatitis - Update 2015. J Hepatol 2015;62:S100-S111. doi:10.1016/j.jhep.2015.03.005

4. Alvarez F, Berg PA, Bianchi FB, et al. International Autoimmune Hepatitis Group Report: review of criteria for diagnosis of autoimmune hepatitis. J Hepatol 1999;31:929-938. doi:10.1016/S01688278(99)80297-9

5. Berger RM, Haworth SG, Bonnet D, et al. FUTURE-2: Results from an open-label, long-term safety and tolerability extension study using the pediatric FormUlation of bosenTan in pUlmonary arterial hypeRtEnsion. Int J Cardiol 2016;202:52-58. doi:10.1016/j. ijcard.2015.08.080

6. Naito A, Terada J, Tanabe N, et al. Autoimmune Hepatitis in a Patient with Pulmonary Arterial Hypertension Treated with Endothelin Receptor Antagonists. Intern Med 2014;53:771-775. doi:10.2169/ internalmedicine.53.1362

7. Klein R, Hintz E, Staehler G. Exacerbation of AIH in a patient with an AIH/systemic sclerosis overlap syndrome and pulmonary arterial hypertension treated with the endothelin-1 receptor antagonist sitaxentan. BMJ Case Rep 2012;2012. doi:10.1136/bcr01-2012-5494

8. Galiè N, Hoeper MM, Gibbs JSR, Simonneau G. Liver toxicity of sitaxentan in pulmonary arterial hypertension. Eur Respir J 2011;37:475-476. doi:10.1183/09031936.00194810

9. Matsunaga N, Kaneko N, Staub AY, et al. Analysis of the Metabolic Pathway of Bosentan and of the Cytotoxicity of Bosentan Metabolites Based on a Quantitative Modeling of Metabolism and Transport in Sandwich-Cultured Human Hepatocytes. Drug Metab Dispos 2016;44:16-27. doi:10.1124/dmd.115.067074

10. Keller S, Karaa A, Paxian M, Clemens MG, Zhang JX. Inhibition of endothelin-1-mediated up-regulation of iNOS by bosentan ameliorates endotoxin-induced liver injury in cirrhosis. Shock 2006;25:306-313. doi:10.1097/01.shk.0000196549.18258.6a 